\title{
Localized breathing modes in granular crystals with defects
}

\author{
G. Theocharis, ${ }^{1}$ M. Kavousanakis, ${ }^{2}$ P. G. Kevrekidis, ${ }^{1}$ Chiara Daraio, ${ }^{3}$ Mason A. Porter, ${ }^{4}$ and I. G. Kevrekidis ${ }^{2}$ \\ ${ }^{1}$ Department of Mathematics and Statistics, University of Massachusetts, Amherst, Massachusetts 01003-4515, USA \\ ${ }^{2}$ Department of Chemical Engineering, Princeton University, Princeton, New Jersey 08544, USA \\ ${ }^{3}$ Graduate Aerospace Laboratories (GALCIT), California Institute of Technology, Pasadena, California 91125, USA \\ ${ }^{4}$ Oxford Centre for Industrial and Applied Mathematics, Mathematical Institute, University of Oxford, Oxford OX1 3LB, United Kingdom
}

(Received 22 June 2009; published 1 December 2009)

\begin{abstract}
We study localized modes in uniform one-dimensional chains of tightly packed and uniaxially compressed elastic beads in the presence of one or two light-mass impurities. For chains composed of beads of the same type, the intrinsic nonlinearity, which is caused by the Hertzian interaction of the beads, appears not to support localized, breathing modes. Consequently, the inclusion of light-mass impurities is crucial for their appearance. By analyzing the problem's linear limit, we identify the system's eigenfrequencies and the linear defect modes. Using continuation techniques, we find the solutions that bifurcate from their linear counterparts and study their linear stability in detail. We observe that the nonlinearity leads to a frequency dependence in the amplitude of the oscillations, a static mutual displacement of the parts of the chain separated by a defect, and for chains with two defects that are not in contact, it induces symmetry-breaking bifurcations.
\end{abstract}

DOI: 10.1103/PhysRevE.80.066601 PACS number(s): 05.45.Yv, 43.25.+y, 45.70. $-\mathrm{n}, 46.40 . \mathrm{Cd}$

\section{INTRODUCTION}

We investigate nonlinear localized modes that result from configurational heterogeneity in granular crystals. Accordingly, our study entails a confluence of three key research themes: intrinsic localization through nonlinearity, wave propagation in granular chains, and extrinsic localization through disorder.

Intrinsic localized modes (ILMs), also known as discrete breathers, have been a central theme for numerous theoretical and experimental studies over the past two decades [1-5]. The original theoretical proposal of ILMs in prototypical settings such as anharmonic nonlinear lattices [6,7] and the rigorous proof of their existence under fairly general conditions [8] motivated numerous studies of such modes in a diverse host of applications, including optical waveguides and photorefractive crystals [9], the denaturation of the DNA double strand [10], micromechanical cantilever arrays [11], nanomechanical resonators [12], superconducting Josephson junctions [13], Bose-Einstein condensation [14], electrical lattices [15], and more. In particular, ILMs seem to play a significant role in biophysics, nonlinear optics, and in condensed matter physics. As shown in Refs. [16], they are expected to contribute not only to the dynamics by also to the thermodynamics of the relevant systems.

One-dimensional (1D) granular crystals, which consist of closely packed chains of elastically interacting particles, have drawn considerable attention during the past few years. This broad interest has arisen from the wealth of available material types or sizes and the ability to tune their dynamic response to encompass linear, weakly nonlinear, and strongly nonlinear regimes [17-20]. Such flexibility makes granular crystals perfect candidates for many engineering applications, including shock and energy absorbing layers [21-24], actuating devices [25], and sound scramblers [26,27]. Because of these possibilities, it is crucial to investigate the effects of defects (imhomogeneities, beads with different masses, etc.), which allow the observation of interesting physical responses such as fragmentation, anomalous reflections, and energy trapping [21-24,28-34].

It is well known from solid-state physics that localized vibrations in (linear) lattices can arise from extrinsic disorder that breaks the discrete translational invariance of a perfect crystal lattice $[35,36]$. Such "disorder" is also well known to introduce interesting wave-scattering effects [37]. This phenomenology arises in a host of physical applications, including superconductors [38], electron-phonon interactions [39], light propagation in dielectric superlattices with embedded defect layers [40], defect modes in photonic crystals [41], and optical waveguide arrays [42-44].

In the present work, we aim to investigate the confluence of the preceding research themes by examining the interplay of "disorder" (which induces localized modes) and nonlinearity in granular crystals. We note in passing that the interaction of impurities with solitary waves or a continuous oscillatory signal in unloaded (i.e., without precompression) monoatomic chains has been investigated both numerically $[28,31]$ and experimentally [45]. In these studies, localized oscillations arise from the interaction of an impurity particle, whose mass is lighter than that of the other particles in an otherwise homogeneous chain, with either a solitary wave or a continuous oscillatory signal. However, these localized oscillations are all transient, fading away as soon as the wave leaves the vicinity of the impurity.

Here, by contrast, we examine long-lived localized breathing oscillations, which form robust nonlinear localized modes (NLMs) induced by the impurities in strongly compressed granular chains. We demonstrate that their frequency depends not only on experimental parameters such as the precompressive force and the constitution (material and size) of the impurity bead but also on the inherent nonlinearity of the system (i.e., on the amplitude of the oscillations). We provide a detailed bifurcation and dynamical analysis of a monoatomic chain with a single lower-mass bead (which we call an "impurity") and extend our considerations to monoatomic chains with a pair of lower-mass impurities. We show that the wave dynamics in the case of nearest-neighbor im- 
purities, which arise in a chain as consecutive lower-mass beads without other intervening particles, differs substantially from that in the case of larger separations between impurities. We focus on the case of next-nearest-neighbor impurities, revealing a rich bifurcation structure in which asymmetric branches of solutions emerge through spontaneous symmetry breaking. We monitor the dynamical manifestation of this bifurcation (and associated instability) and examine how it is affected by a potential initial asymmetry in the impurity masses.

The remainder of our presentation is structured as follows. We start by discussing the Hertzian model of a homogeneous (no impurity) granular crystal. We then perform linear and nonlinear analyses first for monoatomic chains with a single impurity and then for monoatomic chains with a pair of impurities. Finally, we summarize our findings and present some possible future directions.

\section{MONOATOMIC GRANULAR CHAIN}

The interaction between two adjacent elastic spheres is well known to be described by Hertz's law [46]. The relation between the force $F_{0}$ exerted on two identical spheres and the distance $\delta_{0}$ between their centers results from geometric effects and is given by the nonlinear relation

$$
F_{0}=A \delta_{0}^{3 / 2}
$$

where

$$
A=\frac{E \sqrt{2 R}}{3\left(1-\nu^{2}\right)},
$$

$R$ is the radius of the beads, $E$ is the material's elastic (Young's) modulus, and $\nu$ is the Poisson ratio of the bead material.

The dynamics of a 1D chain composed of beads of a single type (i.e., a monoatomic chain) is thus described by the following system of coupled nonlinear ordinary differential equations [17]:

$$
M \ddot{u}_{i}=A\left[\delta_{0}+u_{i-1}-u_{i}\right]_{+}^{3 / 2}-A\left[\delta_{0}+u_{i}-u_{i+1}\right]_{+}^{3 / 2},
$$

where $u_{i}$ is the displacement of the $i$ th bead from its equilibrium position in the initially compressed chain, $i$ $\in\{2, \cdots, N-1\}$, and $M$ is the bead mass. Both ends of the chain are free $\left(u_{0}=u_{1}, u_{N}=u_{N+1}\right)$, subjected to static compression forces $F_{0}$ which are causing the initial displacement $\delta_{0}$ between adjacent particles. The bracket $[s]_{+}$of Eq. (3) takes the value $s$ if $s>0$ and the value 0 if $s \leq 0$, which signifies that adjacent beads are not in contact.

In contrast with Ref. [45], which considered unloaded chains, we investigate strongly precompressed chains, in which $F_{0}$ takes large values. Considering small-amplitude displacements in comparison with the initial ones caused by the precompressive force, so that

$$
\frac{\left|u_{i-1}-u_{i}\right|}{\delta_{0}} \ll 1
$$

implies that one can Taylor expand the forces in a power series. Keeping the displacement terms to fourth order leads to the approximate (" $K_{2}-K_{3}-K_{4}$ ") model

$$
\begin{aligned}
M \ddot{u}_{i}= & K_{2}\left(u_{i+1}-2 u_{i}+u_{i-1}\right)+K_{3}\left[\left(u_{i+1}-u_{i}\right)^{2}-\left(u_{i-1}-u_{i}\right)^{2}\right] \\
& +K_{4}\left[\left(u_{i+1}-u_{i}\right)^{3}+\left(u_{i-1}-u_{i}\right)^{3}\right]
\end{aligned}
$$

where

$$
K_{2}=\frac{3}{2} A \delta_{0}^{1 / 2}, \quad K_{3}=-\frac{3}{8} A \delta_{0}^{-1 / 2}, \quad K_{4}=\frac{3}{48} A \delta_{0}^{-3 / 2} .
$$

The equations of motion (5) are an example of the celebrated Fermi-Pasta-Ulam (FPU) model [47-49]. If we restrict our consideration to very small amplitudes and velocities, then we can neglect all the nonlinear terms from the equations of motion and keep only the harmonic $\left(K_{2}\right)$ term. The spectral band of the ensuing linear chain has an upper cutoff frequency of $\omega_{m}=\sqrt{4 K_{2} / m}$, which corresponds to the lattice vibration in which the neighboring particles oscillate out of phase.

One of the remarkable features of gradually introducing nonlinearity is that its interplay with discreteness leads to the emergence of localized modes even in the absence of any inhomogeneity. Such ILM solutions are generic features of a large class of Hamiltonian lattices that includes the FPU model [4]. Indeed, ILMs have been studied extensively in monoatomic FPU chains [50].

One of the canonical mechanisms for the generation of such nonlinear localized modes is the modulational instability (MI) of the plane wave at the band edge. A detailed analysis of this instability (bifurcation) shows that the MI of the upper cutoff mode manifests itself when the following inequality holds (see Sec. 4.3 of Ref. [4] and references therein):

$$
3 K_{2} K_{4}-4 K_{3}^{2}>0 .
$$

Using Eqs. (6), one can show that Eq. (7) does not hold in our setting. This, in turn, indicates that small-amplitude ILMs bifurcating from the upper band mode do not exist in monoatomic granular crystals. The existence of dark discrete breathers or large-amplitude ILMs remains an interesting open question for future investigation.

\section{MONOATOMIC GRANULAR CHAIN WITH AN IMPURITY}

\section{A. Model}

Consider a 1D monoatomic chain of beads that contains an impurity at the $k$ th site. Suppose that the impurity (the $k$ th bead) is made of the same material as the other particles but has a different radius. In particular, we consider a homogeneous host chain that is composed of spherical beads of nonmagnetic, type 316 stainless steel (which have elastic modulus $E=193 \mathrm{GPa}$, Poisson ratio $\nu=0.3$, and density $\rho$ $=8027.17 \mathrm{~kg} / \mathrm{m}^{3}$ [51]) of radius $R=4.76 \mathrm{~mm}$ and a spherical steel impurity bead with some other radius $r$. We treat the radius of the impurity as a free parameter, though in most cases we will use $r=0.8 R$. We also suppose that the granular chain is compressed by an experimentally accessible static force of $F_{0}=22.25 \mathrm{~N}$.

The presence of the impurity bead in the chain gives rise to a mass defect and leads, in turn, to changes in the force 
constants that destroy the discrete translational symmetry of the crystal. Recalling that the precompressive static force $F_{0}$ induces an initial displacement $\delta_{0}$ between neighboring spheres of the same diameter and defining $\delta_{1}$ to be the displacement that it induces between neighboring spheres of different diameters, the equations of motion (3) at sites $k$ $-1, k$, and $k+1$ become

$$
\begin{gathered}
M \ddot{u}_{k-1}=A_{1}\left[\delta_{0}+u_{k-2}-u_{k-1}\right]_{+}^{3 / 2}-A_{2}\left[\delta_{1}+u_{k-1}-u_{k}\right]_{+}^{3 / 2}, \\
m \ddot{u}_{k}=A_{2}\left[\delta_{1}+u_{k-1}-u_{k}\right]_{+}^{3 / 2}-A_{2}\left[\delta_{1}+u_{k}-u_{k+1}\right]_{+}^{3 / 2}, \\
M \ddot{u}_{k+1}=A_{2}\left[\delta_{1}+u_{k}-u_{k+1}\right]_{+}^{3 / 2}-A_{1}\left[\delta_{0}+u_{k+1}-u_{k+2}\right]_{+}^{3 / 2}, \\
A_{1}=\frac{2 E\left(\frac{R}{2}\right)^{1 / 2}}{3\left(1-\nu^{2}\right)}, \quad A_{2}=\frac{2 E\left(\frac{R r}{R+r}\right)^{1 / 2}}{3\left(1-\nu^{2}\right)},
\end{gathered}
$$

where $m$ and $r$ are the mass and the radius of the impurity bead while $M$ and $R$ denote the mass and the radius of each of the remaining beads.

\section{B. Harmonic potential approximation (linear analysis)}

To understand the underlying linear spectrum of the problem, we linearize Eqs. (3) and (8) about the equilibrium state in the presence of precompression. This yields

$$
\begin{gathered}
m \ddot{u}_{i}=C_{1}\left(u_{i-1}-2 u_{i}+u_{i+1}\right), \quad i \notin\{k-1, k, k+1\}, \\
M \ddot{u}_{k-1}=C_{1}\left(u_{k-2}-u_{k-1}\right)-C_{2}\left(u_{k-1}-u_{k}\right), \\
m \ddot{u}_{k}=C_{2}\left(u_{k-1}-2 u_{k}+u_{k+1}\right), \\
M \ddot{u}_{k+1}=C_{2}\left(u_{k}-u_{k+1}\right)-C_{1}\left(u_{k+1}-u_{k+2}\right), \\
C_{1}=\frac{3}{2} A_{1} \delta_{0}^{1 / 2}, \quad C_{2}=\frac{3}{2} A_{2} \delta_{1}^{1 / 2} .
\end{gathered}
$$

Seeking stationary solutions with frequency $\omega$, we substitute

$$
u_{j}=v_{j} e^{i \omega t}
$$

for all $j$ into Eqs. (9) and obtain the following eigenvalue problem:

$$
\begin{aligned}
& \omega^{2} M\left(\begin{array}{ccccccc}
1 & 0 & & \ldots & & 0 & 0 \\
0 & 1 & & \ldots & & 0 & 0 \\
\vdots & \vdots & \ddots & & & \vdots & \vdots \\
0 & 0 & \ldots & \frac{m}{M} & \ldots & 0 & 0 \\
\vdots & \vdots & & & \ddots & \vdots & \vdots \\
0 & 0 & & \ldots & & 1 & 0 \\
0 & 0 & \ldots & & 0 & 1
\end{array}\right)\left(\begin{array}{c}
v_{1} \\
v_{2} \\
\vdots \\
v_{k} \\
\vdots \\
v_{N-1} \\
v_{N}
\end{array}\right) \\
& =C_{1} \mathbf{V}\left(\begin{array}{c}
v_{2} \\
\vdots \\
v_{k} \\
\vdots \\
v_{N-1} \\
v_{N}
\end{array}\right)
\end{aligned}
$$

where

$$
\begin{array}{r}
\mathbf{V}=\left(\begin{array}{ccccccc}
1 & -1 & 0 & & \ldots & 0 & 0 \\
-1 & 2 & -1 & & \ldots & 0 & 0 \\
\vdots & \vdots & \ddots & & & \vdots & \vdots \\
0 & 0 & \ldots & \mathbf{C} & \ldots & 0 & 0 \\
\vdots & \vdots & & & \ddots & \vdots & \vdots \\
0 & 0 & & \ldots & -1 & 2 & -1 \\
0 & 0 & & \ldots & -1 & 1
\end{array}\right), \\
\mathbf{C}
\end{array}
$$

The eigenvalue problem (11) determines the spectrum of the extended phonon excitations and of the localized defect mode centered at the impurity site. In general, the presence of impurity beads can create two types of vibrational modes:

(1) Resonance modes, when the mass of the impurity bead is larger than the mass of the other beads (i.e., when $m>M)$.

(2) Localized modes, in the opposite case (i.e., when $m<M)$.

Each resonance mode possesses a frequency within the range of frequencies that constitute the phonon band of the homogeneous host crystal and has a vibration amplitude that is larger in the vicinity of the impurity bead. Each localized mode, on the other hand, has a frequency $f_{\text {imp }}$ that lies above the band of the normal-mode frequencies of the homogeneous host crystal and, as shown in Fig. 1, has a vibration amplitude that is large at the impurity site but decreases very rapidly with increasing distance. Reference [45] used 

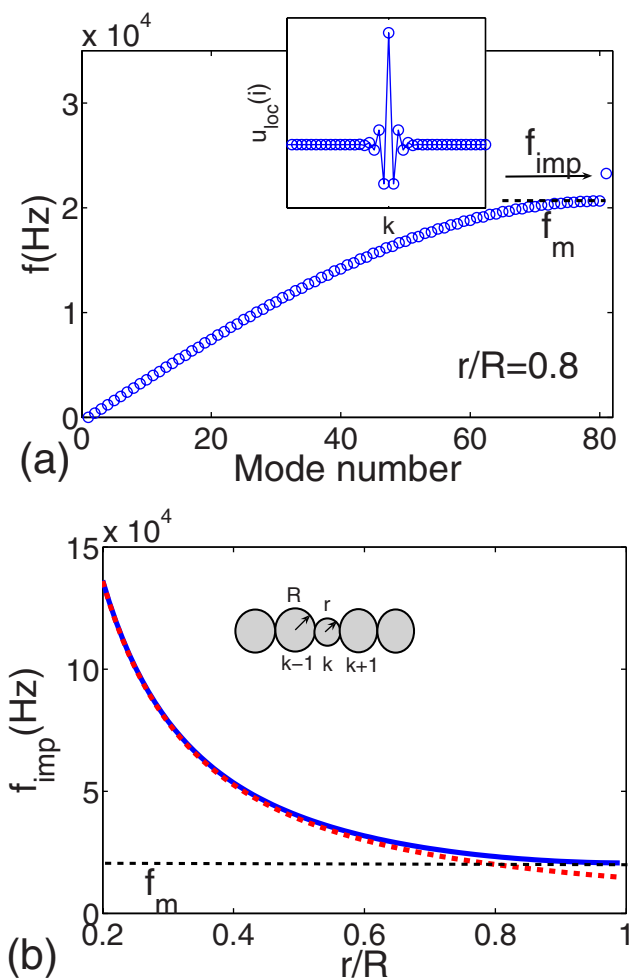

FIG. 1. (Color online) (a) The normal-mode frequencies of the crystal in the presence of the single lighter-mass defect. The presence of the impurity bead leads to the manifestation of a localized mode (see inset) with frequency above the band of the perfect (monoatomic) host crystal. (b) Numerically obtained frequency $f_{i m p}$ (solid curve) of the localized mode as a function of the radius ratio $r / R$ compared with the analytical prediction (dashed curve). As $r / R \rightarrow 1$, one sees that $f_{\text {imp }} \rightarrow f_{m} \approx 20.67 \mathrm{kHz}$ (the horizontal dashed line) and that the deviation between the solid and dashed curves becomes larger.

multiple-scale analysis to obtain the analytical approximation

$$
f_{a} \approx \frac{\sqrt{3}}{2 \pi} \frac{A_{2}^{1 / 3} F_{m}^{1 / 6}}{m^{1 / 2}}
$$

for $f_{i m p}$, where $F_{m}$ is the maximum force magnitude of the solitary wave envelope moves over the defect site. The experimental setup in [45] is quite different from our setting because it has no precompression. As discussed in Ref. [45], the maximum force magnitude of this pulse acts as a "local precompression force" as it travels, which makes the system weakly nonlinear locally and results in localized oscillations at the impurity site. By contrast, in the present setting, the chain is strongly compressed by a static force, which acts globally in a nonlinear fashion and allows localized modes to be maintained indefinitely without further external excitations after they are excited initially (by, e.g., an actuator or the impact of a striker particle). This distinction between local and global forces is the key difference that leads to very long-lived localized oscillations around the impurity bead. Such oscillations can last arbitrarily long in principle, but in laboratory experiments the presence of dissipative effects will eventually result in their attenuation [52].

As illustrated in Fig. 1(b), we find excellent agreement between the analytical expression of Eq. (12) for $F_{m}=F_{0}$, and the frequency of the localized mode obtained by the eigenvalue system (11) up to radii ratios $\frac{r}{R} \approx 0.6$ (see also Fig. 5 of [45]). In particular, for the material parameters and the precompressive force indicated above, an impurity bead of radius $r=0.6 R$ (for which $\frac{m}{M} \approx 0.216$ ) yields a localized mode with frequency $f_{\text {imp }} \approx 31.76 \mathrm{kHz}$, whereas Eq. (12) predicts $f_{a} \approx 30 \mathrm{kHz}$. On the other hand, for $r=0.8 R$ (which yields $\frac{m}{M} \approx 0.512$ ), the eigenvalue system (11) gives $f_{\text {imp }}$ $\approx 23.28 \mathrm{kHz}$ and Eq. (12) predicts $f_{a} \approx 20.03 \mathrm{kHz}$. To provide additional context, we remark that the upper cutoff frequency of the precompressed homogeneous host crystal is given by $f_{m}=\frac{1}{2 \pi} \sqrt{\frac{4 K_{2}}{m}} \approx 20.67 \mathrm{kHz}$. It is clear that the analytical expression in Eq. (12) is expected to be a good approximation only for $m$ sufficiently smaller than $M$. Otherwise, one has to use the numerically obtained frequency $f_{\text {imp }}$. We also note that as the radius of the impurity bead (which is smaller than the regular beads) becomes larger, the difference between the frequency $f_{\text {imp }}$ of the impurity-induced localized mode and the upper cutoff frequency becomes smaller. Indeed, $f_{\text {imp }} \rightarrow f_{m}$ as $r / R \rightarrow 1$ [as shown in Fig. 1(b)], and the localized mode becomes concomitantly more extended.

\section{Continuation and stability analysis}

In the previous section, we examined the linear response of the granular crystal in the presence of a single defect. We now study Eqs. (3) and (8) directly to examine the nonlinear behavior of the chain. From a physical perspective, this allows us to examine the interplay of nonlinearity and "disorder." When a perfect (i.e., homogeneous) nonlinear lattice supports ILMs, the presence of impurities can drastically change the properties of such localized modes. This can result in several interesting phenomena-including the presence of asymmetric impurity modes in nonlinear lattices with a light-mass defect [53] and the existence of stable, nonlinear, heavy-mass impurity modes [54]. As we have mentioned, our nonlinear lattice does not support ILMs [see the inequality (7)], so the aforementioned phenomena are not expected to be present. However, the linear localization at the defect in conjunction with nonlinearity can result in the presence of robust NLMs.

More specifically, it is important to consider whether the nonlinearity of the chain can support the existence of localized modes with frequencies $f$ such that $f_{m}<f<f_{\text {imp. }}$. In the linear limit, the chain does not support vibrations with frequencies in this regime. To answer this question more generally, we perform a parameter continuation in which we start from the linear localized mode and systematically change (in small steps) the frequency from $f_{\text {imp }}$ toward the upper cutoff $f_{m}$. For each of the intermediate frequencies, we identify NLMs to high precision, via a Newton method in phase space, using free boundary conditions and chains with $N=79$ beads. In order to identify the relevant branch of solutions, we use as an initial guess the localized impurityinduced mode [see the inset of Fig. 1(a)], as this was ob- 

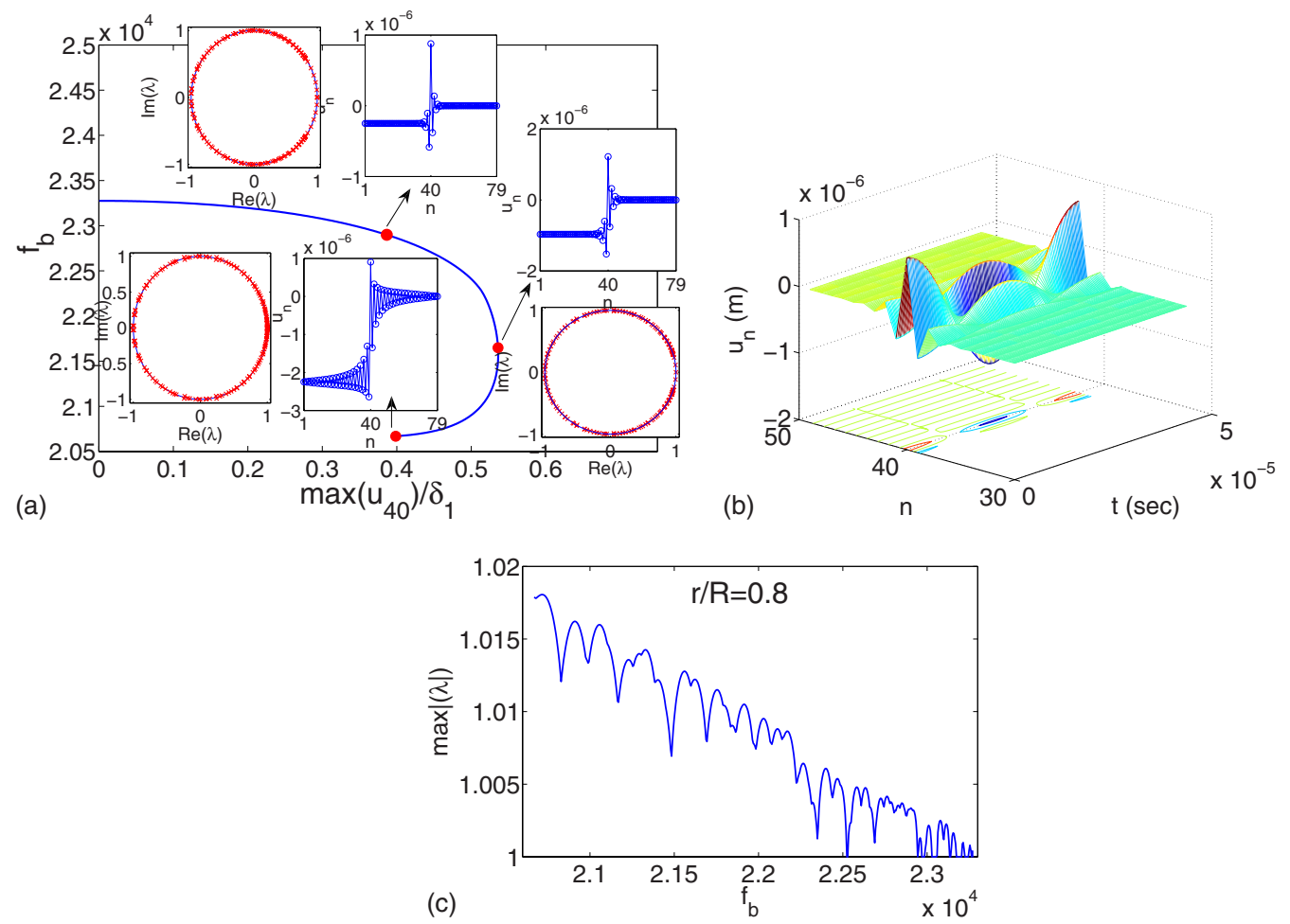

FIG. 2. (Color online) (a) Continuation diagram of the nonlinear impurity modes. Insets: the spatial profiles and the corresponding locations of the Floquet multipliers $\lambda$ in the complex plane of the localized modes with frequencies $f_{1}=22.9 \mathrm{kHz}, f_{2}=21.65 \mathrm{kHz}$, and $f_{m}=20.67 \mathrm{kHz}$. (b) One period of the spatiotemporal evolution of the localized mode with frequency $f_{1}=22.9 \mathrm{kHz}$. (c) The maximum of the absolute value of the Floquet multipliers as a function of the frequency $f_{b}$ of the nonlinear impurity mode.

tained from the linear eigenvalue problem (11). The momenta of all of the sites can be fixed to zero, following [55], due to the time reversibility of the system. For details of this continuation method, see Ref. [4] and references therein.

In Fig. 2, we show the results of the continuation, which allowed us to obtain localized solutions for all frequencies $f \in\left[f_{m}, f_{\text {imp }}\right)$. In insets of Fig. 2(a), we show three examples of these solutions; they have frequencies $f_{1}=22.9 \mathrm{kHz}, f_{2}$ $=21.65 \mathrm{kHz}$, and $f_{m}=20.67 \mathrm{kHz}$. We examined the stability of these localized modes by computing their Floquet multipliers $\lambda_{j}$ to describe the behavior of trajectories near the periodic solution. We show the locations in the complex plane of the Floquet multipliers for the three NLM profiles in insets of Fig. 2(a). As is well known, if all eigenvalues $\lambda_{j}$ have unit magnitude, then the localized periodic solution is linearly stable. However, if $\left|\lambda_{j}\right|>1$ for some $j$, then a perturbation along the corresponding eigenfunction $\mathbf{e}_{\mathbf{j}}$ grows by the factor $\left|\lambda_{j}\right|$ after one period. In Fig. 2(b), we show one period of the spatiotemporal evolution of the localized mode with frequency $f_{1}$. In Fig. 2(c), we show the absolute value of the maximal eigenvalue, as this is associated with the instability growth rate.

We found that the family of the localized solutions exhibits an oscillatory instability. In general, oscillatory instabilities can arise due to the collision of either two Floquet multipliers associated with spatially extended eigenvectors or one multiplier associated with a spatially extended eigenvector and another associated with a spatially localized one. A careful study of the unstable Floquet multipliers and the cor- responding eigenvectors reveals that the oscillatory instabilities are caused by the collision of extended modes belonging to the two arcs of overlapping continuous phonon spectrum of the Floquet matrix [56]. During the continuation of the solutions, typically 3-5 quadruplets of eigenvalues abandon the unit circle after the collision but return to it soon afterward in parameter space. As discussed in Ref. [56], the strength of such instabilities should depend on the system size (i.e., on the number of beads in the chain). In particular, when the size of the system is increased, the magnitude of such instabilities weakens uniformly; simultaneously, the number of such instabilities increases with system size due to the increasing density of colliding eigenvalues. Eventually, these instabilities vanish in the limit of an infinitely large system. We observe that the deviations of the unstable eigenvalues from the unit circle are bounded by 0.02 , and numerical integration of the nonlinear impurity modes up to times of $100 T$ (where $T$ is the period of the corresponding mode) reveals their robustness.

It is relevant to also note that two pairs of the Floquet multipliers are always located at +1 in the complex plane. One of them, often called a "phase mode," describes a rotation of the breather's aggregate phase. The second one arises from the conservation of total mechanical momentum, which is an integral of motion of the nonlinear chains. As one can see in the insets of Fig. 2(a), the spatial profiles of the NLMs have interesting structure. In particular, they are characterized by a kink-shaped distortion of the chain that is caused by the asymmetry in the interaction potential (see Sec. 4.1.4 of [4]). This asymmetry is evident in the $K_{2}-K_{3}-K_{4}$ ap- 

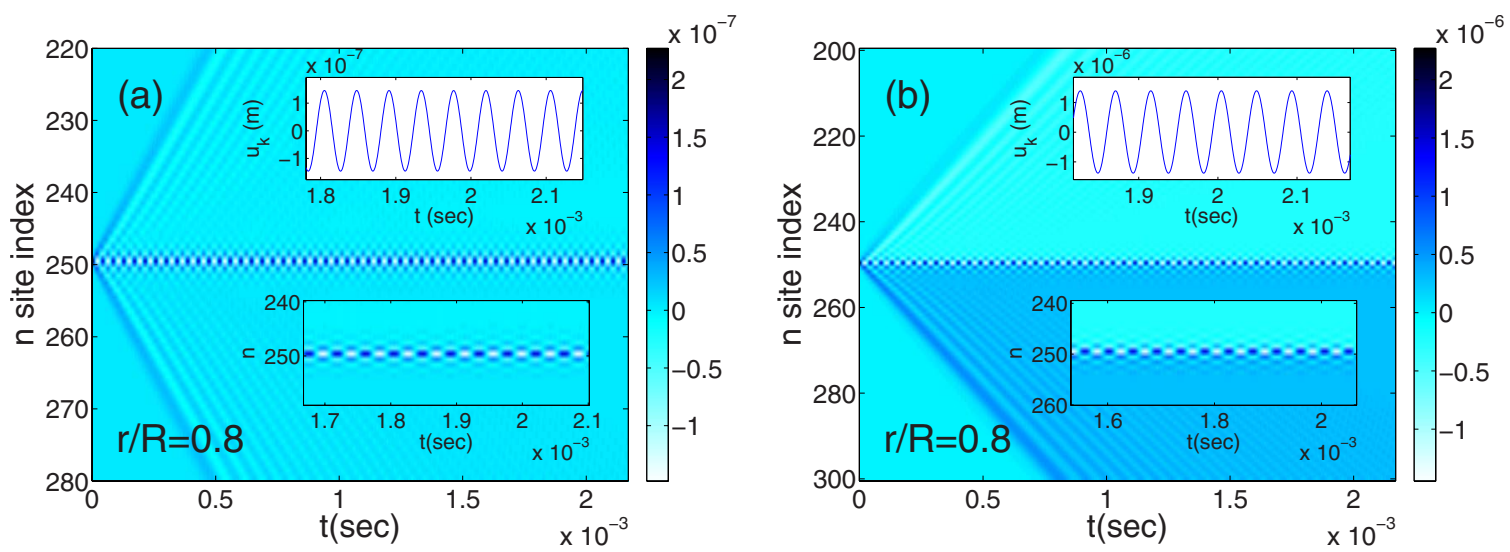

FIG. 3. (Color online) Spatiotemporal evolution of the displacements of the beads with initial conditions $(\mathrm{a}) u_{k}(0)=\delta_{1} / 10$ and $u_{j}=0$ for all $j \neq k$ and (b) $u_{k}(0)=\delta_{1}$ and $u_{j}=0$ for all $j \neq k$. For case (a), the frequency of the oscillations of the impurity site is about $23.25 \mathrm{kHz}$ and $\max \left(u_{k} / \delta_{1}\right) \approx 0.065$, while for case $(\mathrm{b})$, one obtains about $22.47 \mathrm{kHz}$ and $\max \left(u_{k} / \delta_{1}\right) \approx 0.5$. Top insets: the displacements of the impurity bead (which lies at the $k$ th site). Bottom insets: magnifications of the spatiotemporal evolution near the impurity site. Observe that after an initial (transient) stage of energy shedding in the form of sound waves, a nonlinear localized breathing mode forms in the neighborhood of the defect.

proximation of the model, and it arises directly from the fact that $K_{3} \neq 0$. The NLM can be thus viewed as a localized vibration that induces this kind of distortion in the granular crystal. As one approaches the edge of the phonon band (i.e., as $f_{b} \rightarrow f_{m}$ ), the NLMs become more extended and gradually approach their extended (plane wave) linear counterparts at the upper band of the linear spectrum. In this limit, the kinkshaped distortion and the maximum of the absolute values of the Floquet multipliers also increase.

\section{Excitation of nonlinear impurity modes}

In the previous section, we demonstrated that NLMs exist in the gap between the band of phonon modes of the perfect crystal and the localized impurity mode. This demonstrates that their frequency depends not only on the precompressive force and the material parameters of the defect chain (as is the case in the linear limit) but also on the amplitude displacement of the impurity bead-in other words, on the strength of the nonlinearity.

In this light, we showcase in the present section what we believe is the easiest way to observe these NLMs. Our method is based on the use of a simple localized initial excitation. At time $t=0$, we displace the bead at impurity site $k$ by an amount that is strong enough to ensure that the nonlinear terms are no longer negligible in the corresponding equations of motion. Meanwhile, we keep all of the remaining sites at rest. We then integrate the equations of motion (3) and (8) using a fourth-order Runge-Kutta numerical scheme. We expect part of the initially localized energy excitation to spread among the other sites. In order to avoid backscatter of emitted waves, we consider a chain with $N$ $=500$ beads.

We generated long-lived NLMs using two proof-ofprinciple simulations. In the first, we considered a relatively weak initial displacement of the impurity site, $u_{k}(0)=\delta_{1} / 10$, which we show in Fig. 3(a). In the second, we considered a relatively strong initial displacement of the impurity site, $u_{k}(0)=\delta_{1}$, which we show in Fig. 3(b). In both cases, we used the precompression and material parameters discussed previously. Observe that for the strong initial displacement, the nonlinearity causes a substantial distortion of the chain. The frequency of the oscillations of the impurity site was about $23.25 \mathrm{kHz}$ and $\max \left(u_{k} / \delta_{1}\right) \approx 0.065$ for the first simulation. For the second simulation, we observed a frequency of about $22.47 \mathrm{kHz}$ and $\max \left(u_{k} / \delta_{1}\right) \approx 0.5$. Both sets of results are in excellent agreement with the continuation analysis discussed above, which gave frequencies of 23.26 and $22.41 \mathrm{kHz}$ for these particular values of $\max \left(u_{k} / \delta_{1}\right)$. These simulations therefore clearly illustrate the excitation of the previously analyzed NLMs.

\section{MONOATOMIC GRANULAR CHAIN WITH TWO IMPURITIES}

\section{A. Harmonic potential approximation (linear analysis)}

We now consider a crystal with two impurities, which are located at sites $k$ and $l$. As before, we begin with an analysis of the linearized spectrum. We first examine the case of two identical impurities, for which we consider impurity beads made of the same material (stainless steel) as the ones of the host chain (which is again a perfect crystal) but with smaller radius $\left(r_{1}=r_{2}=r=0.8 R\right)$. The left panel of Fig. 4 shows the frequencies that correspond to localized modes, which we obtained using the harmonic approximation, as a function of the distance between the impurities. Interestingly, for this value of radius ratio, when the impurity beads are in contact $(l-k=1)$, the phonon spectrum has just a single localized mode with frequency $f_{\text {imp }} \approx 25.28 \mathrm{kHz}$. As shown in inset (a) in the left panel of Fig. 4, the corresponding mode is antisymmetric. By examining the phonon spectrum for the case $l-k=1$ as a function of the radius ratio $r / R$, we found that for $r / R<0.8$ a symmetric mode leaves the phonon band and becomes progressively more localized as the radius ratio is decreased. In particular, for $r / R=0.4$ the phonon spectrum 

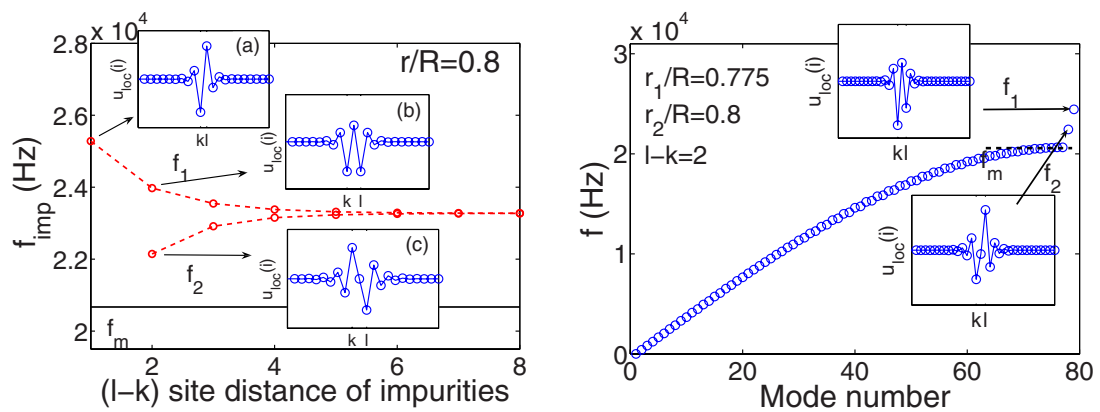

FIG. 4. (Color online) Left panel: the frequencies of the localized modes generated by two identical impurities as a function of the distance (number of sites) between them. The insets show corresponding localized modes for (a) $l-k=1$, for which the impurities are in contact and $[(\mathrm{b}),(\mathrm{c})] l-k=2$. As the impurities are placed increasingly far apart, we see that $f_{1}-f_{2} \rightarrow 0$. Right panel: the normal-mode frequencies of a granular crystal with two impurities of different radii $\left(r_{1}=0.775 R\right.$ and $\left.r_{2}=0.8 R\right)$ and $l-k=2$.

contains two localized modes: an antisymmetric one with $f_{1} \approx 62.26 \mathrm{kHz}$ and a symmetric one with $f_{2} \approx 38.48 \mathrm{kHz}$. When $l-k \geq 2$, there are two localized modes even for $r / R$ $=0.8$. In particular, for $l-k=2$, the frequency of the symmetric mode [inset (b) in the left panel of Fig. 4] is $f_{1} \approx 23.98 \mathrm{kHz}$, and the frequency of the antisymmetric mode [inset (c) in the left panel of Fig. 4] is $f_{2} \approx 22.14 \mathrm{kHz}$. As the two impurities are placed farther apart, $f_{1}-f_{2} \rightarrow 0$ and $f_{1}, f_{2} \rightarrow f_{\text {imp }} \approx 23.28 \mathrm{kHz}$, which is the frequency of a single-impurity localized mode (see the discussion in the previous section).

We now consider the phonon spectrum for the case of two different impurities with separation $l-k=2$ and bead radii $r_{1}=0.775 R$ and $r_{2}=0.8 R$. (As before, the impurity beads are made of the same material as those in the host chain.) As one can see by comparing the insets in the left and right panels of Fig. 4, even a slight difference in the radii of impurity beads results in an asymmetric modification of the corresponding localized modes. In this case, we also obtain slightly larger mode frequencies: $f_{1} \approx 24.45 \mathrm{kHz}$ and $f_{2} \approx 22.44 \mathrm{kHz}$.

\section{B. Continuation and stability analysis for two identical impurities}

We show the results of our parameter continuation for the case of two identical impurity beads in contact $(l-k=1)$ with radius ratio $r / R=0.8$ in Fig. 5 . We find essentially the same phenomenology as we obtained for granular crystals with a single impurity. That is, we obtain a family of weakly oscillatory, unstable localized solutions due to finite size effects. The magnitudes of the deviations of the unstable eigenvalues from the unit circle are smaller than 0.025. Again as before, the modes become wider and the characteristic kink-shaped distortion of the chain becomes larger as one approaches the frequency $f_{m}$. We show three examples of this family of localized solutions (with frequencies $f_{1}=24.75 \mathrm{kHz}$, $f_{2}=22.55 \mathrm{kHz}$, and $f_{m}=20.65 \mathrm{kHz}$ ) in the insets of Fig. $5(\mathrm{a})$. In the rest of the insets, we show the locations in the complex plane of their corresponding Floquet multipliers.

Now consider a granular crystal with two impurities that are not in contact. More specifically, we focus on the prototypical case of $l-k=2$ and $r / R=0.8$. As indicated above, the corresponding phonon spectrum contains two localized modes: a symmetric one, shown in inset (b) of the left panel of Fig. 4, and an antisymmetric one, shown in inset (c) of the left panel of Fig. 4. In Fig. 6, we show continuation diagrams, which display the frequencies of NLMs in terms of the maximum displacement of one of the impurity beads ( $l=41$, in a chain with $N=79$ beads) normalized to the characteristic (precompression-induced) displacement $\delta_{1}$. First, we examine the family of solutions that arises from the symmetric linear mode at $f_{1} \approx 23.98 \mathrm{kHz}$ [see Fig. 6(a)]. Stability analysis demonstrates the presence of a weak oscillatory instability, which we also observed for a single impurity and a pair of in-contact impurities. Now consider the NLMs that bifurcate from the antisymmetric linear mode, for which
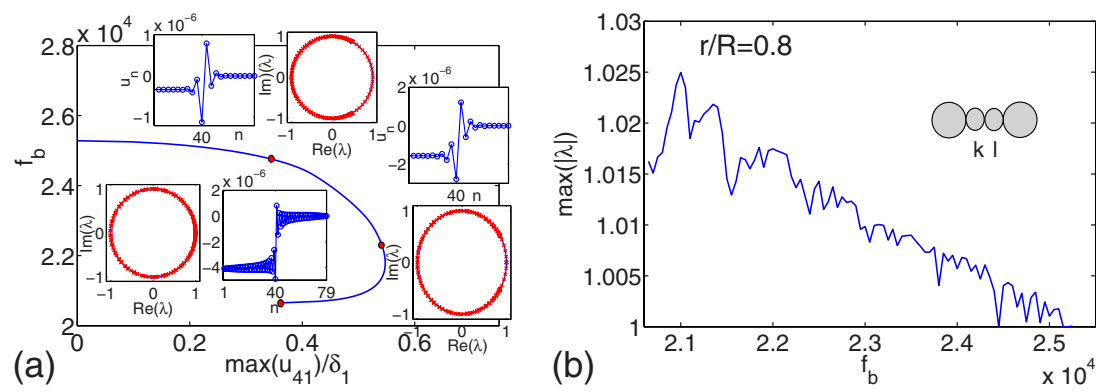

FIG. 5. (Color online) (a) Continuation diagram of the nonlinear impurity modes for the case of two identical impurity beads in contact $(l-k=1)$. One set of insets shows the profiles of the localized modes with frequencies $f_{1}=24.75 \mathrm{kHz}, f_{2}=22.55 \mathrm{kHz}$, and $f_{m}=20.65 \mathrm{kHz}$, and the other set shows the corresponding locations in the complex plane of their Floquet multipliers $\lambda$. (b) The maximum of the absolute values of the Floquet multipliers as a function of the frequency $f_{b}$ of the nonlinear impurity mode. 

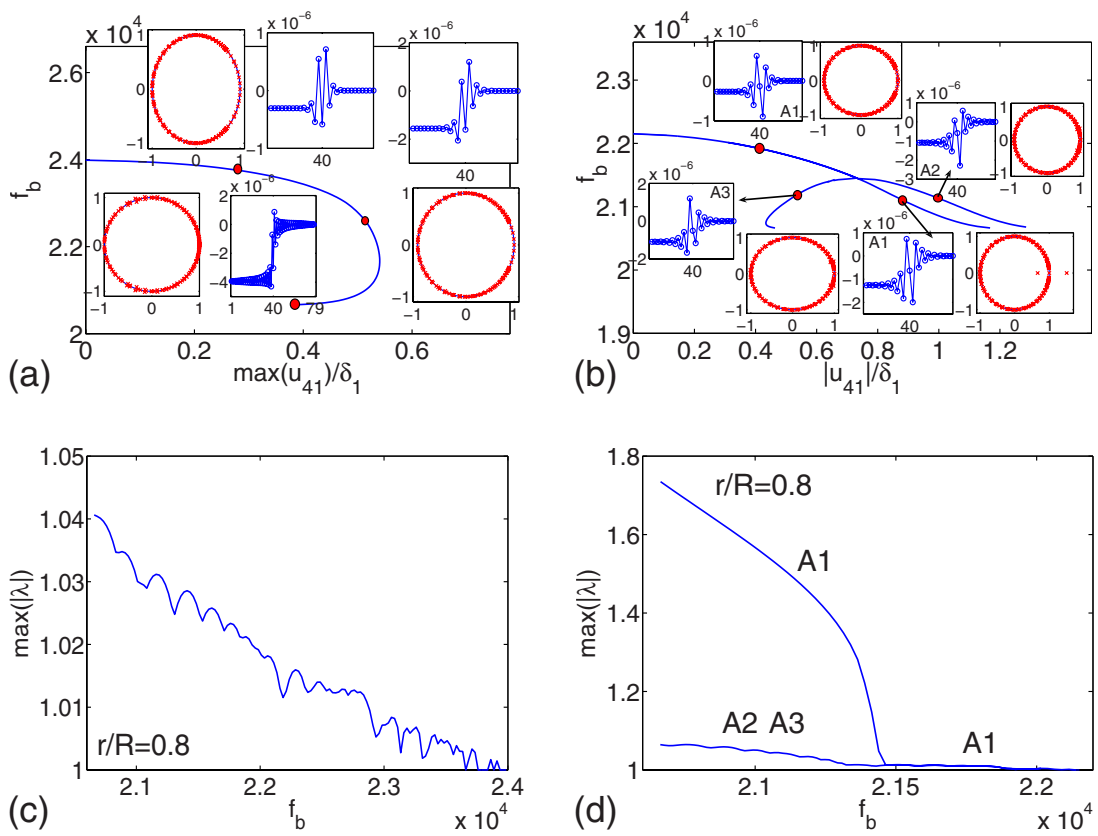

FIG. 6. (Color online) (a) Continuation diagram for the nonlinear impurity modes that originate from the symmetric linear mode of a granular crystal with two impurities that are separated by one bead (i.e., $l-k=2$ ). One set of insets shows the profiles for the localized modes with frequencies $f=23.68 \mathrm{kHz}, f=22.26 \mathrm{kHz}$, and $f=20.66 \mathrm{kHz}$. The other set shows the locations in the complex plane of their corresponding Floquet multipliers $\lambda$. (b) Continuation diagram for the antisymmetric solution branch $\left(A_{1}\right)$, showing the generation of asymmetric branches $\left(A_{2}\right.$ and $\left.A_{3}\right)$ that originate from the bifurcation at $f \approx 21.44 \mathrm{kHz}$. The insets show the wave profiles (and their Floquet multipliers) of the antisymmetric localized mode before the bifurcation $\left(f_{b}=21.94 \mathrm{kHz}\right)$, the antisymmetric mode after the bifurcation $\left(f_{b}=21.11 \mathrm{kHz}\right)$, and the asymmetric mode with frequency $f_{b}=21.11 \mathrm{kHz}$. (c) The maximum of the absolute values of the Floquet multipliers in terms of the frequency of the nonlinear impurity mode $f_{b}$ for the symmetric branch. (d) Same as panel (c), but for the antisymmetric $\left(A_{1}\right)$ and asymmetric $\left(A_{2}, A_{3}\right)$ branches.

$f_{2} \approx 22.14 \mathrm{kHz}$. This family of solutions, which corresponds to the branch $A_{1}$ of the continuation diagram in panel (b) of Fig. 6, is initially weakly unstable (due to the finite-size effects discussed previously). At $f \approx 21.56 \mathrm{kHz}$, a pair of Floquet multipliers leaves the phonon bands. The corresponding eigenmodes are symmetric and become progressively more localized as the frequency decreases [57]. At $f \approx 21.44 \mathrm{kHz}$, these two localized modes collide at the $(+1,0)$ point on the unit circle, giving rise to a strong instability (called a "harmonic instability") that is connected to a bifurcation of the corresponding NLM. Two new families of solutions (branches $A_{2}$ and $A_{3}$ ) emerge from this bifurcation. Aside from the kink-shaped distortion of the system, these families are symmetric with respect to each other and weakly unstable due to finite-size effects. Thus, this bifurcation is somewhat reminiscent of a pitchfork bifurcation [58]. In the case of the newly formed branches past the bifurcation point (i.e., $A_{2}$ and $A_{3}$ ), and particularly at $f \approx 21.34 \mathrm{kHz}$, the localized pair of eigenmodes that has formed enters the band of eigenvalues associated with extended perturbations, giving rise to a new oscillatory instability. Although this kind of instability is size-dependent, it persists even in the limit of an infinite system [59] (in contrast to the oscillatory instability caused by the collision of two extended modes).

It is worth noting that the setting of granular chains with two next-nearest-neighbor impurities (i.e., with $l-k=2$ ) is reminiscent of double-well configurations in other contexts. For example, both defocusing and focusing nonlinear
Schrödinger (NLS) equations with double-well potentials are known to exhibit "symmetry breaking" bifurcations like the one discussed above [60]. (The defocusing case is relevant to the present setting.) Such bifurcations have even been observed experimentally in both optical [61] and atomic systems [62].

Examining the temporal dynamics of the unstable antisymmetric mode evinces the symmetry-breaking phenomenon. To trigger the relevant instability, we use the sum of the unstable solution with $f=21.214 \mathrm{kHz}$ and the corresponding unstable localized eigenfunction of the linearization around the solution as an initial condition in the full nonlinear equations of motion (3) and (8). Its dynamical evolution, which we show in Fig. 7(a), reveals the "symmetry breaking" at $t \approx 0.4 \mathrm{~ms}$. This is followed by alternating oscillations between the two impurity sites (corresponding to the asymmetric modes on $A_{2}$ and $A_{3}$ ). As illustrated in Fig. 7 (b), the dynamical evolution of the weak oscillatory instability of the asymmetric modes is somewhat similar. As we pointed out above, such dynamics is reminiscent of theoretical [60] and experimental [61] observations of the instability manifestation in NLS equations with double-well potentials.

\section{Continuation and stability analysis for two distinct impurities}

As we discussed above, we observe a pitchfork-like bifurcation, indicating the emergence of two families of asymmet- 

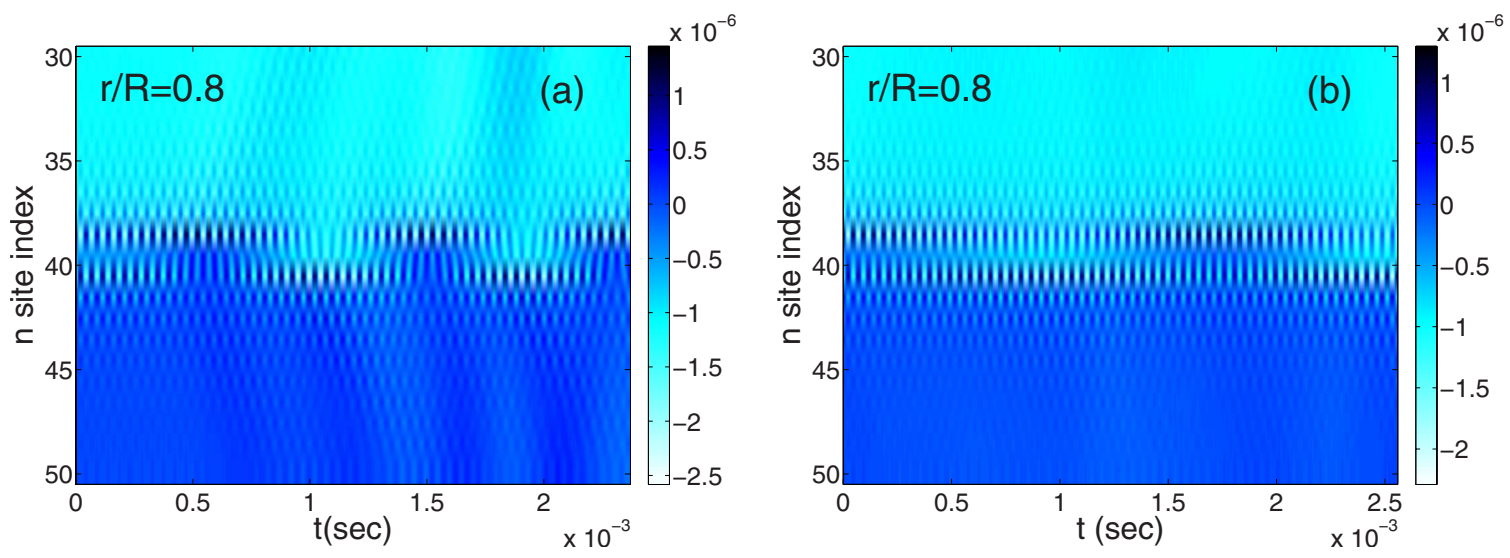

FIG. 7. (Color online) Spatiotemporal evolution of the bead displacements for (a) the harmonically unstable antisymmetric localized mode with $f_{b}=21.214 \mathrm{kHz}$ (for which $\max |\lambda| \approx 1.437$ ) and (b) for the weakly oscillatory unstable asymmetric localized mode with the same frequency (for which $\max |\lambda| \approx 1.032$ ).

ric localized solutions if the two impurities in the granular chain are identical. Employing the aforementioned analogy with NLS equations, this implies that the observed dynamics corresponds to that obtained in a symmetric "double well" that can be construed as having been induced by the identical, next-nearest-neighbor impurities. A natural generalization is to consider how such phenomenology changes when these impurities are distinct. In this case, the "double well" becomes asymmetric, and (from bifurcation theory) one expects to see something analogous to what is sometimes called an imperfect pitchfork bifurcation [58]. Such a scenario, which has been observed in NLS equations with asymmetric double wells [63], involves an asymmetric perturbation of the pitchfork structure, resulting in a saddle-node bifurcation and an isolated branch of solutions.

To observe this breakdown and obtain the associated bifurcation picture, we consider the case of two distinct impurities with slightly different radii (namely, $r_{1}=0.775 R$ and $r_{2}=0.8 R$ ) on the prototypical next-nearest-neighbor case of $l-k=2$. Recall that we showed the normal-mode frequencies for this configuration in the right panel of Fig. 4. We show the continuation and the stability diagrams for the family of solutions originating from $f_{2} \approx 22.44 \mathrm{kHz}$ in Fig. 8. (We omit the continuation of the solutions that emerge from the linear mode with frequency $f_{1}$ because it resembles the one shown in the left panel of Fig. 6(a).) As suggested above, this amounts to a saddle-node bifurcation. The branches of solutions that are analogous to the $A_{1}$ and $A_{3}$ branches from Fig. 6 collide at a critical value $f \approx 21.1 \mathrm{kHz}$ and disappear. Linear stability analysis shows that one colliding branch consists of strongly harmonically unstable solutions and that the other consists of weakly oscillatory unstable solutions. The isolated branch, which manifests itself because we have broken the perfect pitchfork and arises from the mode with frequency $f_{2}$ in the linear limit, is only weakly unstable instead of exhibiting the strong instability we showed in Fig. 6. Once again, this is reminiscent of the NLS phenomenology observed in Ref. [63].

\section{CONCLUSIONS AND FUTURE CHALLENGES}

In conclusion, we have investigated the formation of localized modes due to the interplay of nonlinearity and disorder (i.e., the presence of defects) in granular crystals. While
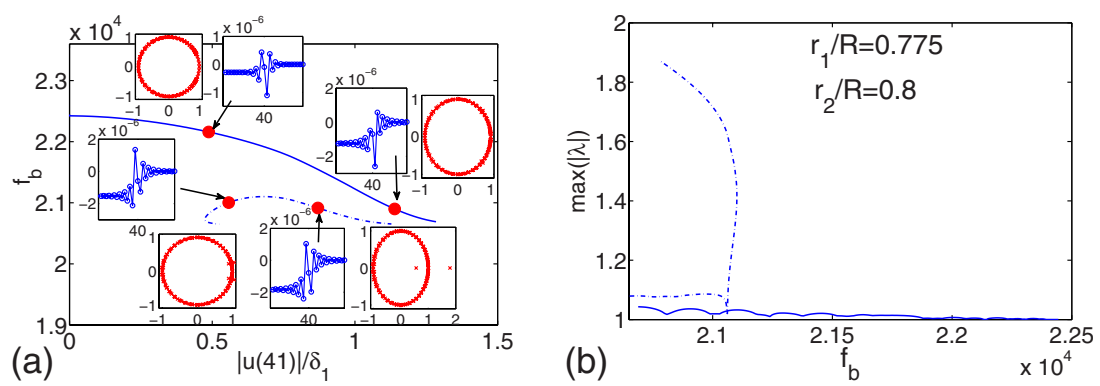

FIG. 8. (Color online) (a) Continuation diagram for the nonlinear impurity mode that originates from the linear mode with $f_{2} \approx 22.44 \mathrm{kHz}$ for the case of two distinct impurities with slightly different radii (namely, $r_{1}=0.775 R$ and $r_{2}=0.8 R$ ) on the prototypical next-nearest-neighbor case of $l-k=2$. This diagram also shows two additional families of solutions that collide at $f \approx 21.1 \mathrm{kHz}$. The insets show the spatial profiles (and associated Floquet multipliers $\lambda$ ) of the localized modes at $f_{b}=22.16 \mathrm{kHz}$ and $f_{b}=20.9 \mathrm{kHz}$. In the latter case, we show an example from each branch. (b) The maximum of the absolute values of the Floquet multipliers as a function of the frequency of the nonlinear impurity mode $f_{b}$ for the different branches. Observe that the isolated branch that stems from the linear impurity mode is only weakly unstable in the frequency interval that we have probed, whereas the two branches that arise via the saddle-node bifurcation and exist for $f<21.1 \mathrm{kHz}$ encompass both a strongly unstable family of solutions and a weakly unstable one. 
previous research has been dedicated to the computational and experimental identification of such modes $[18,45]$, the present work identifies such modes in a numerically exact (up to a prescribed tolerance) form and offer a systematic analysis of their linear stability. We have argued that these localized modes are likely to be absent in monoatomic chains, and we have illustrated that the inclusion of even a single defect can produce a linear defect mode whose interplay with nonlinearity generates a whole family of localized modes. We have demonstrated that these waves are robust, and it should be straightforward to generate them in experiments for a wide range of initial conditions. We extended our single-defect investigation to multiple-defect settings, for which we examined the prototypical situation of crystals with two defect sites. We identified a much richer phenomenology in such situations, as we observed families of both near-symmetric and near-antisymmetric states. We subsequently demonstrated that the latter yields additional families of asymmetric solutions through pitchfork-like bifurcations, which we analyzed for defect pairs containing both identical and distinct impurities.

Although this paper presents some of the first steps toward a systematic understanding and classification of localized breathing modes in granular crystals, there are numerous interesting future directions that should be pursued. First, one can consider progressively larger numbers of defects. By analogy with NLS settings [64], we expect this to reveal not only a wealth of additional phenomenology (e.g., new families of waves and more complicated bifurcation structures) but also implications for how things progress toward the infinite-defect limit. If all defects occur only as next-nearestneighbors, we would obtain a perfect diatomic (two species) crystal in this limit. Such a crystal is naturally expected to support intrinsic localized mode solutions in the band gap between its acoustic and optical bands. Indeed, our preliminary results indicate that such gap breather solutions do arise. The detailed examination of such modes, and their higher-dimensional generalizations (including possibly gap vortex modes [65]), are among our future goals.

A different but related problem is the study of ILMs in the equilibrium configurations of statically and strongly compressed chains, which consist of rigid elements that are coupled by torsional springs [66]. The coupled torsional springs of the type discussed in Ref. [66] are appropriately modeled by the discrete sine-Gordon equation, whose timeindependent form leads to the standard map analyzed in that work in some detail. Because of the local term in the nonlinearity (which is absent here), the linear spectrum is bounded, so that $\omega_{\min }<\omega_{\text {linear }}<\omega_{\max }$. By contrast, in a granular crystal, one instead has $0<\omega_{\text {linear }}<\omega_{m}$. In the discrete sine-Gordon case, the general theory of bifurcations of such ILMs [4] illustrates that such modes will exist even in a homogeneous lattice (i.e., one without any defects); they bifurcate from the lower edge of the spectrum (i.e., from $\omega_{\text {min }}$ ) and exist for frequencies $\omega<\omega_{\min }$. This feature is notably absent in a granular crystal (where $\left.\omega_{\min }=0\right)$. Note, however, that should a defect (e.g., via a different mass or spring coupling) be inserted such that a linear frequency $\omega_{\text {imp }}>\omega_{\max }$ arises, then a nonlinear defect mode would be expected to emerge in the sine-Gordon lattice just as in the granular crystal analyzed herein. The similarities and differences between these systems would be an interesting area for future investigations.

\section{ACKNOWLEDGMENTS}

P.G.K. gratefully acknowledges support from NSF through Grants No. NSF-DMS-0349023 (CAREER) and No. NSF-DMS-0806762, as well as from the Alexander von Humboldt Foundation. The work of IGM and MK was partially supported by NSF and ACS(PRF). C.D. thanks NSF CAREER and NSF Grant No. CMMI-0825345 for support of this project.
[1] D. K. Campbell, S. Flach, and Yu. S. Kivshar, Phys. Today 57(1), 43 (2004).

[2] S. Flach and C. R. Willis, Phys. Rep. 295, 181 (1998).

[3] R. S. MacKay, Physica A 288, 174 (2000).

[4] S. Flach and A. V. Gorbach, Phys. Rep. 467, 1 (2008).

[5] S. Aubry, Physica D 103, 201 (1997).

[6] A. J. Sievers and S. Takeno, Phys. Rev. Lett. 61, 970 (1988).

[7] J. B. Page, Phys. Rev. B 41, 7835 (1990).

[8] R. S. MacKay and S. Aubry, Nonlinearity 7, 1623 (1994).

[9] Y. S. Kivshar and G. P. Agrawal, Optical Solitons: From Fibers to Photonic Crystals (Academic Press, San Diego, CA, 2003).

[10] M. Peyrard, Nonlinearity 17, R1 (2004).

[11] M. Sato, B. E. Hubbard, and A. J. Sievers, Rev. Mod. Phys. 78, 137 (2006).

[12] E. Kenig, B. A. Malomed, M. C. Cross, and R. Lifshitz, Phys. Rev. E 80, 046202 (2009)

[13] J. J. Mazo and T. P. Orlando, Chaos 13, 733 (2003).

[14] O. Morsch and M. Oberthaler, Rev. Mod. Phys. 78, 179
(2006).

[15] L. Q. English, R. B. Thakur, and R. Stearrett, Phys. Rev. E 77, 066601 (2008).

[16] G. P. Tsironis and S. Aubry, Phys. Rev. Lett. 77, 5225 (1996); A. Bikaki, N. K. Voulgarakis, S. Aubry, and G. P. Tsironis, Phys. Rev. E 59, 1234 (1999); R. Reigada, A. Sarmiento, and K. Lindenberg, ibid. 64, 066608 (2001).

[17] V. F. Nesterenko, Dynamics of Heterogeneous Materials (Springer-Verlag, New York, NY, 2001).

[18] S. Sen, J. Hong, J. Bang, E. Avalos, and R. Doney, Phys. Rep. 462, 21 (2008).

[19] C. Daraio, V. F. Nesterenko, E. B. Herbold, and S. Jin, Phys. Rev. E 73, 026610 (2006).

[20] C. Coste, E. Falcon, and S. Fauve, Phys. Rev. E 56, 6104 (1997).

[21] C. Daraio, V. F. Nesterenko, E. B. Herbold, and S. Jin, Phys. Rev. Lett. 96, 058002 (2006).

[22] J. Hong, Phys. Rev. Lett. 94, 108001 (2005). 
[23] F. Fraternali, M. A. Porter, and C. Daraio, Mech. Adv. Mat. Struct. (to be published).

[24] R. Doney and S. Sen, Phys. Rev. Lett. 97, 155502 (2006).

[25] D. Khatri, C. Daraio, and P. Rizzo, Proc. SPIE 6934, 69340U (2008).

[26] C. Daraio, V. F. Nesterenko, E. B. Herbold, and S. Jin, Phys. Rev. E 72, 016603 (2005).

[27] V. F. Nesterenko, C. Daraio, E. B. Herbold, and S. Jin, Phys. Rev. Lett. 95, 158702 (2005).

[28] E. Hascoet and H. J. Hermann, Eur. Phys. J. B 14, 183 (2000).

[29] E. J. Hinch and S. Saint-Jean, Proc. R. Soc. London, Ser. A 455, 3201 (1999).

[30] J. B. Hong and A. G. Xu, Appl. Phys. Lett. 81, 4868 (2002).

[31] S. Sen, M. Manciu, and J. D. Wright, Phys. Rev. E 57, 2386 (1998).

[32] M. Manciu, S. Sen, and A. J. Hurd, Physica A 274, 588 (1999); Physica A 274, 607 (1999).

[33] M. A. Porter, C. Daraio, E. B. Herbold, I. Szelengowicz, and P. G. Kevrekidis, Phys. Rev. E 77, 015601(R) (2008).

[34] M. A. Porter, C. Daraio, I. Szelengowicz, E. B. Herbold, and P. G. Kevrekidis, Physica D 238, 666 (2009).

[35] A. A. Maradudin, E. W. Montroll, and G. H. Weiss, Theory of Lattice Dynamics in the Harmonic Approximation (Academic, New York, 1963).

[36] I. M. Lifshitz, Nuovo Cimento 3, 716 (1956) ; I. M. Lifshitz and A. M. Kosevich, Rep. Prog. Phys. 29, 217 (1966).

[37] A. A. Maradudin, Theoretical and Experimental Aspects of the Effects of Point Defects and Disorder on the Vibrations of Crystal (Academic Press, New York, 1966).

[38] A. F. Andreev, JETP Lett. 46, 584 (1987); A. V. Balatsky, Nature (London) 403, 717 (2000).

[39] M. I. Molina and G. P. Tsironis, Phys. Rev. B 47, 15330 (1993); G. P. Tsironis, M. I. Molina, and D. Hennig, Phys. Rev. E 50, 2365 (1994).

[40] E. Lidorikis, K. Busch, Q. Li, C. T. Chan, and C. M. Soukoulis, Phys. Rev. B 56, 15090 (1997).

[41] S. Y. Lin, E. Chow, V. Hietala, P. R. Villeneuve, and J. D. Joannopoulos, Science 282, 274 (1998); M. G. Khazhinsky and A. R. McGurn, Phys. Lett. A 237, 175 (1998).

[42] U. Peschel, R. Morandotti, J. S. Aitchison, H. S. Eisenberg, and Y. Silberberg, Appl. Phys. Lett. 75, 1348 (1999).

[43] R. Morandotti, H. S. Eisenberg, D. Mandelik, Y. Silberberg, D. Modotto, M. Sorel, C. R. Stanley, and J. S. Aitchison, Opt. Lett. 28, 834 (2003).

[44] E. Smirnov, C. E. Rüter, M. Stepić, V. Shandarov, and D. Kip,
Opt. Express 14, 11248 (2006).

[45] S. Job, F. Santibanez, F. Tapia, and F. Melo, Phys. Rev. E 80, 025602(R) (2009).

[46] L. D. Landau and E. M. Lifshitz, Theory of Elasticity (Pergamon, Oxford, 1986).

[47] D. K. Campbell, P. Rosenau, and G. Zaslavsky, Chaos 15, 015101 (2005)

[48] M. A. Porter, N. J. Zabusky, B. Hu, and D. K. Campbell, Am. Sci. 97, 214 (2009).

[49] G. Huang and B. Hu, Phys. Rev. B 57, 5746 (1998).

[50] S. Flach and A. Gorbach, Chaos 15, 015112 (2005); B Sanchez-Rey, G. James, J. Cuevas, and J. F. R. Archilla, Phys. Rev. B 70, 014301 (2004).

[51] http://www.efunda.com

[52] R. Carretero-Gonzalez, D. Khatri, M. A. Porter, P. G. Kevrekidis, and C. Daraio, Phys. Rev. Lett. 102, 024102 (2009).

[53] A. S. Kovalev, F. Zhang, and Y. S. Kivshar, Phys. Rev. B 51, 3218 (1995).

[54] Y. S. Kivshar, F. Zhang, and A. S. Kovalev, Phys. Rev. B 55, 14265 (1997).

[55] J. L. Marín and S. Aubry, Nonlinearity 9, 1501 (1996).

[56] J. L. Marín and S. Aubry, Physica D 119, 163 (1998).

[57] C. Baesens, S. Kim, and R. S. MacKay, Physica D 113, 242 (1998).

[58] G. Iooss and D. D. Joseph, Elementary Stability and Bifurcation Theory (Springer, New York, 1980).

[59] M. Johansson and Y. S. Kivshar, Phys. Rev. Lett. 82, 85 (1999).

[60] R. K. Jackson and M. I. Weinstein, J. Stat. Phys. 116, 881 (2004); D. Ananikian and T. Bergeman, Phys. Rev. A 73, 013604 (2006).

[61] P. G. Kevrekidis, Z. Chen, B. A. Malomed, D. J. Frantzeskakis, and M. I. Weinstein, Phys. Lett. A 340, 275 (2005).

[62] M. Albiez, R. Gati, J. Fölling, S. Hunsmann, M. Cristiani, and M. K. Oberthaler, Phys. Rev. Lett. 95, 010402 (2005).

[63] G. Theocharis, P. G. Kevrekidis, D. J. Frantzeskakis, and P. Schmelcher, Phys. Rev. E 74, 056608 (2006).

[64] T. Kapitula, P. G. Kevrekidis, and Z. Chen, SIAM J. Appl. Dyn. Syst. 5, 598 (2006).

[65] E. A. Ostrovskaya and Yu. S. Kivshar, Phys. Rev. Lett. 93, 160405 (2004); D. Song, C. Lou, L. Tang, X. Wang, W. Li, X. Chen, K. J. H. Law, H. Susanto, P. G. Kevrekidis, J. Xu, and Z. Chen, Opt. Express 16, 10110 (2008).

[66] G. Domokos and P. J. Holmes, J. Nonlinear Sci. 3, 109 (1993). 